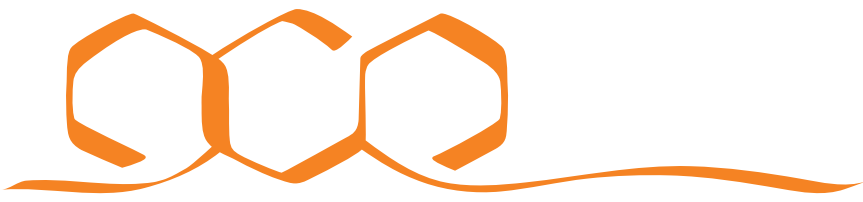 \\ COMMUNICATIONS CHEMISTRY
}

ARTICLE

https://doi.org/10.1038/s42004-019-0192-6

OPEN

Corrected: Publisher Correction

\section{Dual wettability on diarylethene microcrystalline surface mimicking a termite wing}

\author{
Ryo Nishimura (10 1, Kengo Hyodo', Hiroyuki Mayama (1) ${ }^{2}$, Satoshi Yokojima ${ }^{3,4}$, Shinichiro Nakamura (i) ${ }^{4} \&$ \\ Kingo Uchida (1) ${ }^{1,4}$
}

The termite wing has a specific property of wetting in contact with a water droplet: it adsorbs water mist, whereas larger water droplets are bounced on the surface. This is owing to the survival strategy of termites. Here, we reproduce the termite wing's dual wettability by a photoinduced crystal growth technique. Upon UV irradiation to a microcrystalline surface of a mixture of two diarylethenes, two types of needle-shaped crystals of distinctly different sizes are observed to grow. The surface shows behavior akin to the termite wing's dual wettability. The bouncing ability of a water droplet is attributed to the smaller-sized needle crystals, whereas the adhesive property is owing to the larger-sized ones, explaining the microstructures of the termite wing. Considering dissipation energy and adhesion energy, the bouncing ability and dual wettability can be explained theoretically. The surface could potentially be used in water harvesting applications.

\footnotetext{
${ }^{1}$ Department of Materials Chemistry, Ryukoku University, Seta, Otsu, Shiga 520-2194, Japan. ${ }^{2}$ Department of Chemistry, Asahikawa Medical University, 2-11-1 Midorigaoka-higashi, Asahikawa, Hokkaido 078-8510, Japan. ${ }^{3}$ School of Pharmacy, Tokyo University of Pharmacy and Life Sciences, 1432-1 Horinouchi, Hachioji, Tokyo 192-0392, Japan. ${ }^{4}$ RIKEN Cluster for Science, Technology and Innovation Hub, Nakamura Laboratory, 2-1 Hirosawa, Wako, Saitama 3510198, Japan. Correspondence and requests for materials should be addressed to H.M. (email: mayama@asahikawa-med.ac.jp)

or to K.U. (email: uchida@rins.ryukoku.ac.jp)
} 
$\mathrm{n}$ nature, many plants and insects have specifically structured surfaces that show specific surface properties. The unique micro- and nanostructures on such surfaces can be seen in the self-cleaning effect of lotus leaves ${ }^{1}$, the superhydrophobic forces exerted by a water strider's leg$^{2}$, the attachment mechanism of geckos $^{3}$, the structure colors of the peacock ${ }^{4}$, or morpho butterfly ${ }^{5,6}$, and many other natural phenomena ${ }^{7-12}$. These structures have received much attention, and artificial systems have been fabricated by mimicking such natural surfaces. Water repellence supports the performance of self-cleaning materials, and it has been studied in both natural and artificial systems ${ }^{12}$. Historically, the most important finding is the "lotus effect" of Barthlott and Neinhuis ${ }^{13}$. The superwater-repellent and selfcleaning effects of the lotus leaf have been attributed to the double-roughness structure of surfaces with micro- and nanostructures (trichomes, cuticular folds, and wax crystals), as well as to the hydrophobic properties of the epicuticular wax ${ }^{14}$.

Recently, it was reported that many terrestrial insects use nonwetting surfaces to reduce the risks of living in environments with much rain and other water surfaces that the insect may encounter ${ }^{15-20}$. Watson et al. ${ }^{18}$ reported that the complex structure of the termite's wing surface shows specific wettability (Supplementary Fig. 1) ${ }^{19}$. The termite is one of the social insects, and it is known that high humidity is important for their breeding. One of the termites Nasutitermes sp. and Microcerotermes $s p$. fly during the rain period to avoid attacks by predators, whereas water is essential for building nests and soil tunnels, i.e., moist soil is necessary for burrowing ${ }^{18,19}$. Accordingly, they have delicate structures, or "dual wettability", on their wings, and these specific structures work to minimize interaction with water bodies at various length scales to effectively reduce the body mass ${ }^{18,19}$. The termite's wing surface is covered with two types of projections of different sizes (Supplementary Fig. 1). One is a hair-like projection (macrotrichia) that is $\sim 50 \mu \mathrm{m}$ long and 1 $\mu \mathrm{m}$ wide, whereas the other is a star-shaped projection (micrasters) of 5-6 $\mu \mathrm{m}$ in height and width. These hairs protect the wing membrane from the contact with large water droplets, whereas the small projections work to keep the small droplets on the surface of the wing ${ }^{18,19}$. The small droplets gather to build up large ones and are finally removed via the hair arrays. Furthermore, the approximation of body weight after wetting suggested the importance of collection ability by micraster. The estimated additional body weight relation to the total body weight of Nasutitermes sp. and Microcerotermes sp. are several \%. In fact, larger droplets were repelled by hair-like structures, whereas small droplets were collected by star-shaped structures ${ }^{18}$. Watson et al. ${ }^{18}$ argued the Nasutitermes sp. and Microcerotermes sp. have water-collecting ability, and owing to the ability, they can control their flight. Interestingly, the termites that fly during rainy period have hair structures on their wings, but other termites have no hair shaped structures on their wings (Supplementary Figs. 2-4). Owing to these functions, the termite can minimize interaction with micro-sized water droplets while flying in rain or even storm.

Consequently, termite wings have a structure that forms the foundation of their survival strategy. Increasingly, sustainability is also important in human society and the management of water, particularly regarding the important issues of the environment and energy. We believe that better understanding the structure of termite wings would provide great hints for material design as an essential component of humidity management in human society. And to build it is to understand. Therefore, this research attempts to fabricate functional material replicating termite wing from diarylethene.

Diarylethene derivatives are well-known compounds whose photochromic properties, such as the thermal stability of both isomers, and high photoreactivity even in the crystalline states, are excellent among the photochromes. These unique properties are indispensable to the current research, and we applied them to the design of photoinduced topographical control systems ${ }^{21-27}$. In previous papers, we showed that surface topography could be structured by using a crystal growth technique (CGT) ${ }^{27}$ of photogenerated closed-ring isomer $1 \mathrm{c}$ of thermally stable photochromic diarylethene $\mathbf{1}$ (Fig. 1) ${ }^{21}$. Upon UV irradiation to the microcrystalline film of 10 (Fig. 1), the needle-shaped crystals of 1c grew on the surface. Then, a superhydrophobic property was observed. Moreover, we can switch this property by melting these crystals by irradiation with visible light, thus causing the superhydrophobicity to disappear. In the next step, we mimicked the double-roughness structured surface of the lotus leaf and reproduced it on the microcrystalline surface of 1o. Consequently, we succeeded in demonstrating the water droplet-bouncing phenomena as observed for the lotus leaf in nature ${ }^{25}$. The photoinduced topographical changes of $\mathbf{2 0}$ were similar to those of $\mathbf{1 0}$, whereas the size of the needle-shaped crystal of $2 \mathrm{c}$ was much smaller than that of $1 \mathbf{c}$, when they grew at $30^{\circ} \mathrm{C}^{23,24}$. We took advantage of this difference in the current study.

Here we prepare a photo-responsive superhydrophobic surface using a mixture of diarylethenes 10 and 2o. The mixture of two types of diarylethenes may produce the surface with the sum of two different characteristics or completely different characteristics. The obtained photogenerated rough surface consisting of the two different sized needle-shaped crystals $1 \mathrm{c}$ and $2 \mathrm{c}$ shows specific bouncing ability mimicking a termite wing as we have intended. Then, we analyze the surface structures and clarified the relation between the wettability by monitoring the different sizes of water droplet on the surfaces.
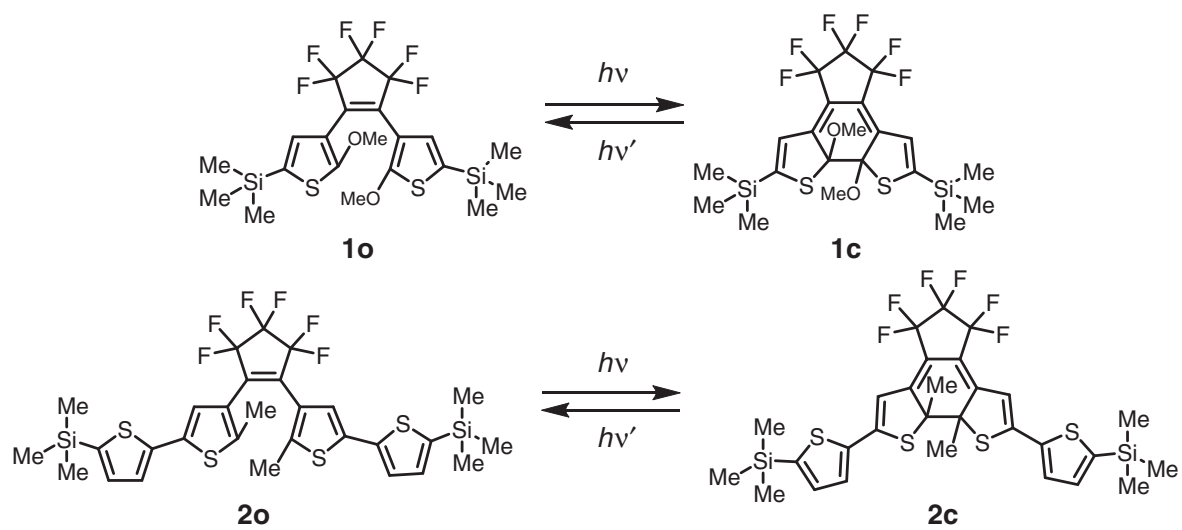

Fig. 1 Molecular structures of diarylethenes. Molecular structures and photoisomerization of diarylethenes $\mathbf{1}$ and $\mathbf{2}$ 


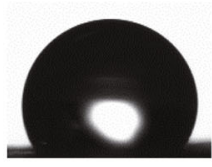

$C A: 122.8 \pm 2.0$

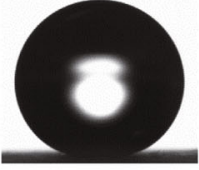

$C A: 163.7 \pm 0.9^{\circ}$
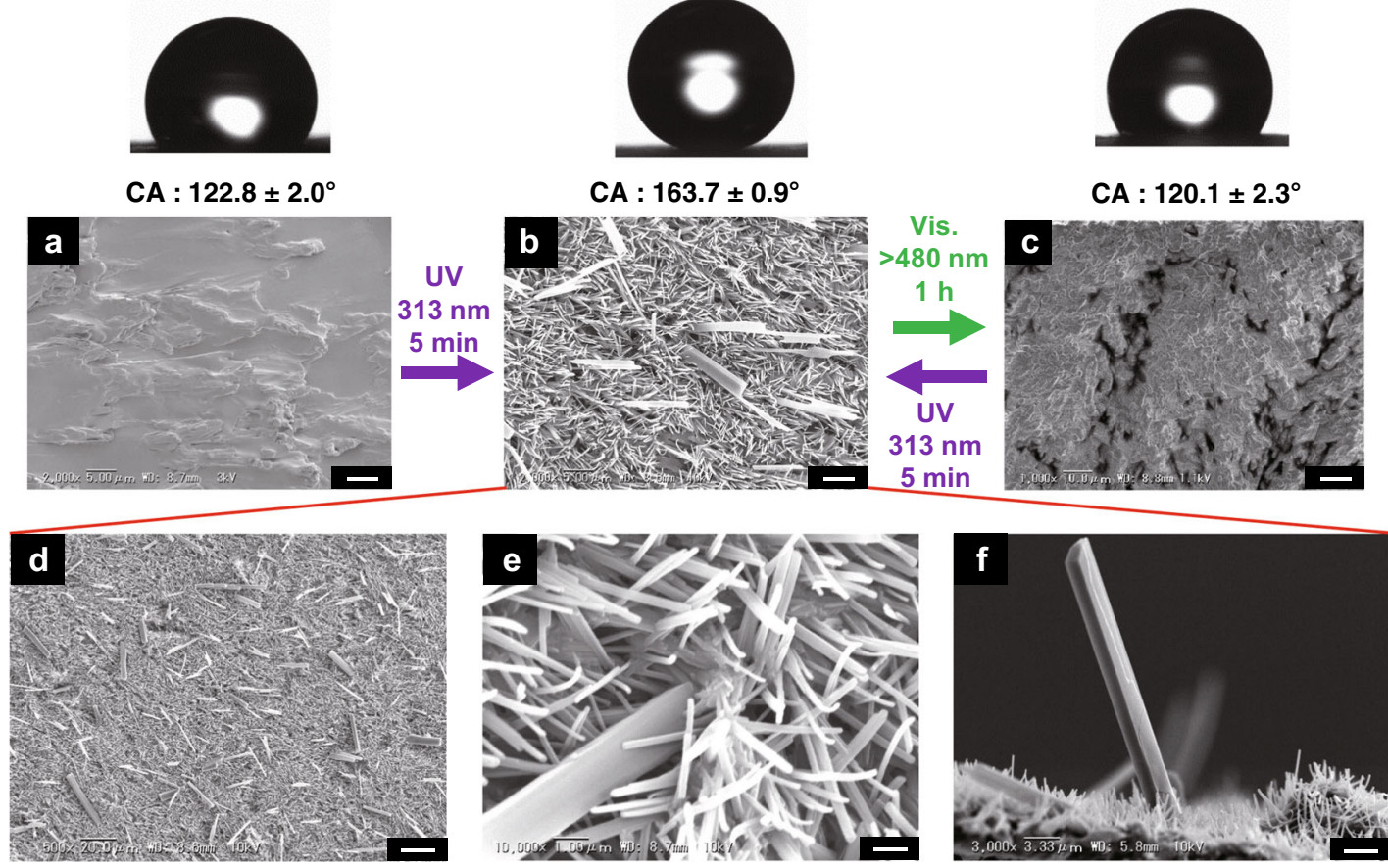

Fig. 2 Wettability and reversible crystal growths on the surface. Images of a water droplet (Volume: $1.5 \mu \mathrm{L}$ ) on rough surfaces and SEM images a-f of topographical changes of microcrystalline films made from a mixture of $\mathbf{1 0}$ and $\mathbf{2 0}$ (molecular ratio, 10:20=1:1) by alternating irradiation with UV and visible light. a Before UV irradiation; b surface UV $(313 \mathrm{~nm})$ light irradiation for 5 min followed by storage at $30^{\circ} \mathrm{C}$ in the dark for 9 days; $\mathbf{c}$ visible $(\lambda>$ $480 \mathrm{~nm}$ ) light irradiation for $1 \mathrm{~h}$ while kept at $80^{\circ} \mathrm{C}$. Scale bars: $5.00 \mu \mathrm{m}$ for $\mathbf{a}, \mathbf{b}, \mathbf{c} ; 20.0 \mu \mathrm{m}$ for $\mathbf{d} ; 1.00 \mu \mathrm{m}$ for $\mathbf{e}, 3.33 \mu \mathrm{m}$ for $\mathbf{f}$

\section{Results}

Preparation of crystalline films mimicking the termite wing. To examine whether the surface of the mixture of $\mathbf{1 0}$ and 20 possess the properties of termite wing as we have expected, we first investigated its properties. A solution containing equimolar amount of 10 and 20 was coated onto a glass plate. After the solvent was evaporated in vacuo, the surface of the microcrystalline film was covered with plate-like crystals (Fig. 2a). On the initial surface, the contact angle (CA) of a water droplet was only $123^{\circ}$. The crystallinity of mixed microcrystalline surface was confirmed by XRD measurement (Supplementary Fig. 5). Then, the surface was irradiated for $5 \mathrm{~min}$ with $313-\mathrm{nm}$ light followed by storage at $30^{\circ} \mathrm{C}$, which is higher than the $T_{\mathrm{g}}$ of both 10 and 20 crystals, as crystal growths of $1 \mathrm{c}$ and $2 \mathrm{c}$ were observed on a softened surface above $T_{\mathrm{g}}$ (Supplementary Fig. 9c) ${ }^{26}$. After 9 days' storage at this temperature in the dark, the surface was covered with two types of needle-shaped crystals of 1c and 2c (Surf $\mathbf{1 c}_{\mathbf{c}+\mathbf{2}}$, Fig. 2b and Supplementary Figs. 6, 15). SEM images at different scales of angles are shown in Fig. 2d-f. By comparing the scanning electron microscope (SEM) images of three types of surfaces, the larger needle-shaped crystals grown on the mixed microcrystalline surface are crystals of 1c, and their lengths and widths are about 16 and $1.5 \mu \mathrm{m}$, respectively, whereas the smaller ones are crystals of $2 \mathrm{c}$ with lengths and widths of $\sim 1.9$ and $0.2 \mu \mathrm{m}$, respectively (Supplementary Fig. 7). The intervals between the crystals were estimated from SEM images. We estimate the number of crystals per unit area as $12.6 \mathrm{~mm}^{-2}$ and $5.00 \times$ $10^{6} \mathrm{~mm}^{-2}$ for 1c and 2c, respectively (Supplementary Fig. 8). These sizes are quite consistent with those observed for homomicrocrystalline surfaces (Fig. 3). The activation energies of the crystal growth were measured independently in the previous work (1c: $143 \mathrm{~kJ} / \mathrm{mol}, 2 \mathrm{c}: 58 \mathrm{~kJ} / \mathrm{mol}$ ). Low activation energy of $2 \mathrm{c}$ compared with 1c probably reflect the low crystallinity of $2 \mathrm{c}$, resulting the bulky molecular structure. The surface was prepared with good reproducibility. Upon visible $(\lambda>480 \mathrm{~nm})$ light irradiation for $1 \mathrm{~h}$ at $80^{\circ} \mathrm{C}$, the needle-shaped crystals were melted (Fig. 2c). The formed structures were very similar, in spite of the difference in the ratio, indicating that the crystal growth of the needle-shaped crystals of $1 c$ and $2 c$ did not affect each other. To confirm this, we attempted to prepare such surfaces by changing the ratio of 10 and 2o (Supplementary Fig. 11); in fact, the surface topography is not so sensitive to the molecular ratio of $\mathbf{1 0}$ and 20. The CA of a water droplet changed with the surface topographies. On the initial surface (Fig. 2a), the CA was only $123^{\circ}$. Owing to the needle-shaped crystal growth of $\mathbf{1 c}$ and $2 \mathrm{c}$ on the surface, CA increased to $164^{\circ}$ upon irradiation with UV light to Surf $\mathbf{1 c}_{\mathbf{1}+\mathbf{2 c}}$. The dynamic CAs of a water droplet were also observed. The advancing and receding contact angles $\left(\mathrm{CA}_{\mathrm{ad}}\right.$ and $\left.\mathrm{CA}_{\mathrm{rec}}\right)$ were $164.2 \pm 1.2^{\circ}$ and $159.6 \pm 0.6^{\circ}$, respectively, and sliding angle (SA) was $2^{\circ}$. Upon visible light irradiation at $80^{\circ} \mathrm{C}$, CA decreased to $120^{\circ}$. By alternate irradiation with $\mathrm{UV}$ and visible light, the surface topography, along with $\mathrm{CA}$, changed reversibly at least three times (Supplementary Fig. 12).

As we reported in previous papers, the larger crystals $(\sim 5-10 \mu \mathrm{m}$ in diameter and $20-30 \mu \mathrm{m}$ in length) of 1c grown by Ostwald ripening were apt to show water-adhesive properties $^{22}$, whereas the smaller sub-micro meter crystals $(0.2-0.3 \mu \mathrm{m}$ in diameter and $2.2-2.5 \mu \mathrm{m}$ in length) of $2 \mathrm{c}$ showed superwaterrepellency ${ }^{23,24}$.

Water dropping test. In the current case, the microcrystalline surface consists of only 10 forming a rough surface of larger needle-shaped crystals with water-adhesive properties (Surf Ic $_{\text {, }}$ Fig. 4e, Supplementary Movie 2), whereas the microcrystalline surface consists of only 20 forming a rough surface of smaller needle-shaped crystals with water-bouncing properties $\left(\mathbf{S u r f}_{\mathbf{2 c}}\right.$, 


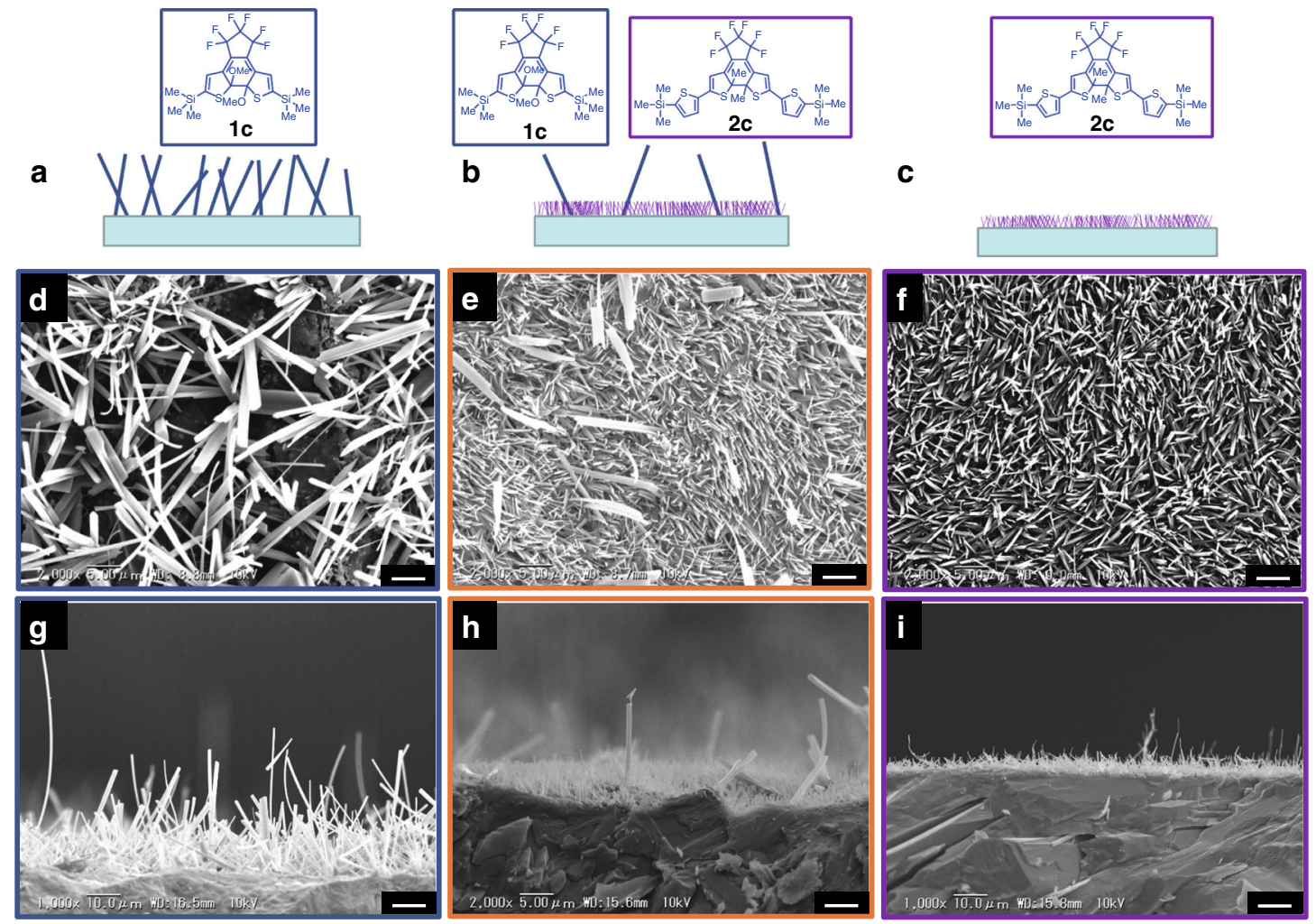

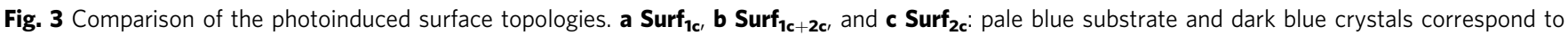
crystals of open- and closed-ring isomers, respectively. SEM images of each surface; $\mathbf{d}$ and $\mathbf{g}$ : surface with needle-shaped crystals of $\mathbf{1 c}$, $\mathbf{e}$ and $\mathbf{h}$ : surface with needle-shaped crystals of $\mathbf{1 c}$ and $\mathbf{2 c}$, $\mathbf{f}$ and $\mathbf{i}$ : surface with needle-shaped crystals of $\mathbf{2 c}$. Scale bars: $5 \mu \mathrm{m}$ for $\mathbf{d}-\mathbf{f}$ and $\mathbf{h}$ images, which are magnified 2000 times; $10 \mu \mathrm{m}$ for $\mathbf{g}$ and $\mathbf{i}$ images, which are magnified 1000 times

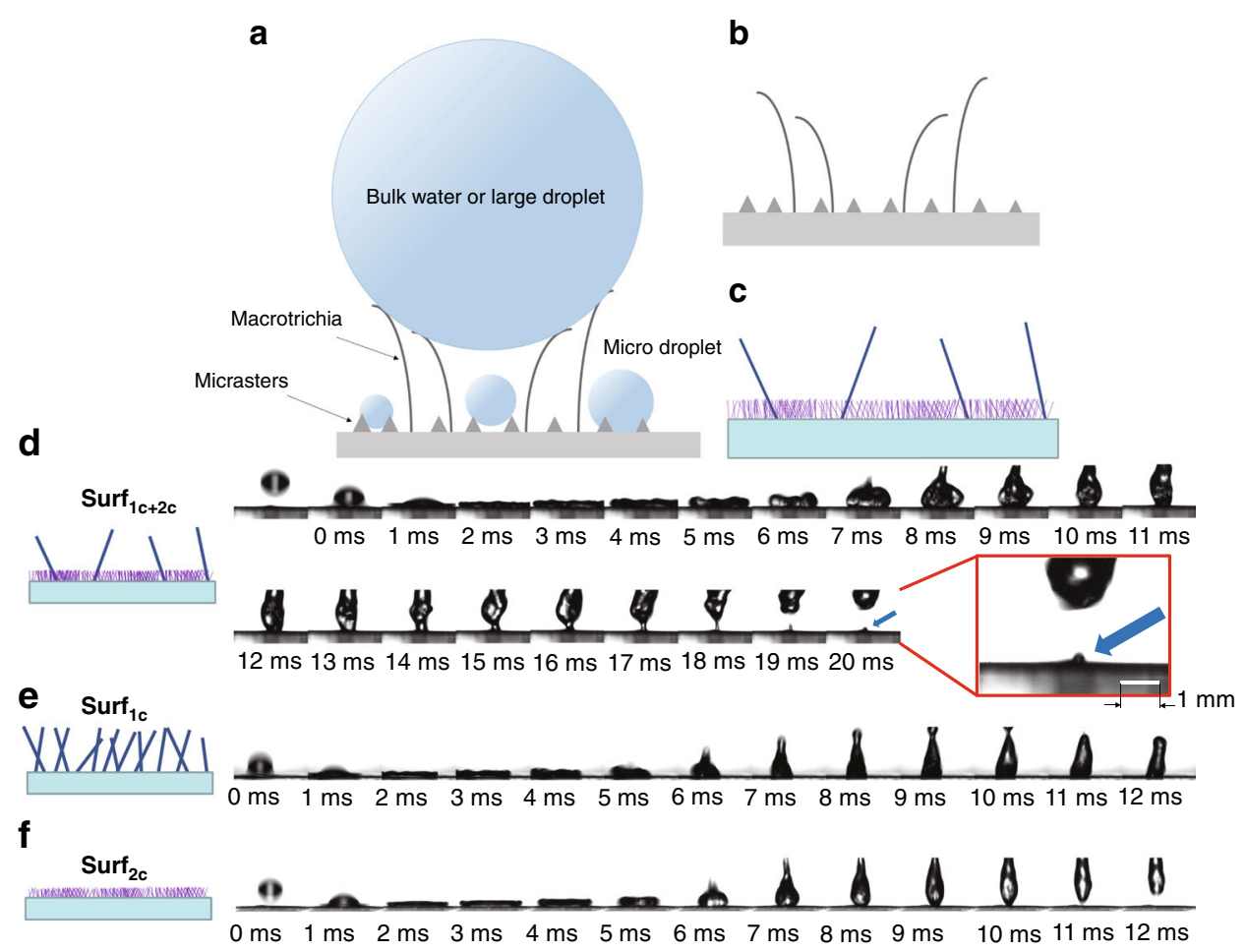

Fig. 4 Illustration of the surface structures and bouncing phenomena. a Schematic illustration of water droplet on the termite wing. b Schematic illustration of surface structures of the termite wing. c Schematic illustration of the surface structures mimicking the surface structures of the termite wing. Water droplets $(7.6 \pm 0.6 \mu \mathrm{L})$ were poured from a height of $10 \mathrm{~cm}$ onto the surfaces for monitoring the bouncing phenomena. $\mathbf{d}$ Optical images of a bouncing

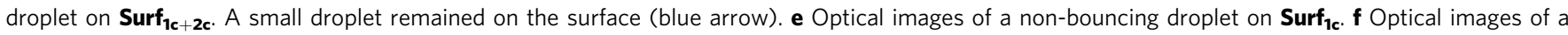
bouncing droplet on Surf $_{\mathbf{2 c}}$. Scale bar of expanded image is $1 \mathrm{~mm}$ 
Fig. 4f, Supplementary Movie 3). The essential point of the current study is to simultaneously grow the two types of crystals of different sizes. Consequently, we could achieve wateradhesive and -bouncing properties on the same surface.

Unlike static CAs and SAs, the dynamic surface properties are different from those of natural superhydrophobic surfaces (e.g., the surface of a lotus leaf). Water droplet-bouncing experiments were performed by releasing water from a height of $10 \mathrm{~cm}$ above the three types of superhydrophobic surfaces $\left(\right.$ Surf $_{1 \mathrm{c}}$, Surf $_{2 \mathrm{c}}$, and Surf $\mathbf{1 c}_{\mathbf{c}+\mathbf{2 c}}$ ) (Fig. 4, Supplementary Movies 1-3). We released a water droplet to Surf $\mathbf{1}_{\mathbf{c}+\mathbf{2} \text { c }}$ from a height of $10 \mathrm{~cm}$, and a small portion of water remained on the surface (Fig. 4d, Supplementary Movie 1). On this surface, either needle-shaped crystals worked to provide either water adhesion or repellency. Then, we released water droplets onto Surf $\mathbf{I c}_{\mathbf{1 c}}$ and Surf $_{2 c}$. On Surf ${ }_{1 c}$, water droplets were adsorbed onto the surface (Supplementary Movie 2), whereas on Surf 2 , water droplets bounced (Supplementary Movie 3). These results indicated that on Surf $\mathbf{1 c}_{\mathbf{1 c}+\mathbf{2} \text { c }}$, the longer needle-shaped crystal of 1c achieved water-capturing capability, whereas the smaller needle-shaped crystals of $2 \mathrm{c}$ achieved water-repelling capability.
Water spraying test. From the above discussion, Surf $\mathbf{1 c}_{1 \mathrm{c}+2 \mathrm{c}}$ thus has the dual capabilities of capturing and repelling water owing to the presence of the two types of needle-shaped crystals of different sizes. This kind of wettability is the same as that of a termite wing, even though the small (large) needle-shaped crystals repel (capture) a droplet, unlike the role of the hairs and micrasters of termite wings, where the small micrasters capture droplets but the large hairs repel them. On the surface of termite wing, by spraying the microdroplet $(20-150 \mu \mathrm{m})$, small-sized droplets $(<100 \mu \mathrm{m})$ were maintained on the wing surface $^{18}$. In order to regenerate the functions of the termite wing, we also sprayed microdroplet $(20-640 \mu \mathrm{m})$ onto Surf $\mathbf{1 c}_{\mathbf{c}}$, Surf $_{2 c}$, and Surf $1 \mathrm{c}+\mathbf{2 c}$. In fact, we could observe microdroplets bouncing on the surfaces (Supplementary Movie 4). We monitored the bouncing phenomena with a high-speed camera (Fig. 4). Consequently, we could observe two cases: microdroplets showing bouncing and non-bouncing phenomena (Fig. 5).

We measured the sizes of all droplets contacting the surfaces and summarized the results as a distribution. By comparing the distributions based on the size of the droplets,
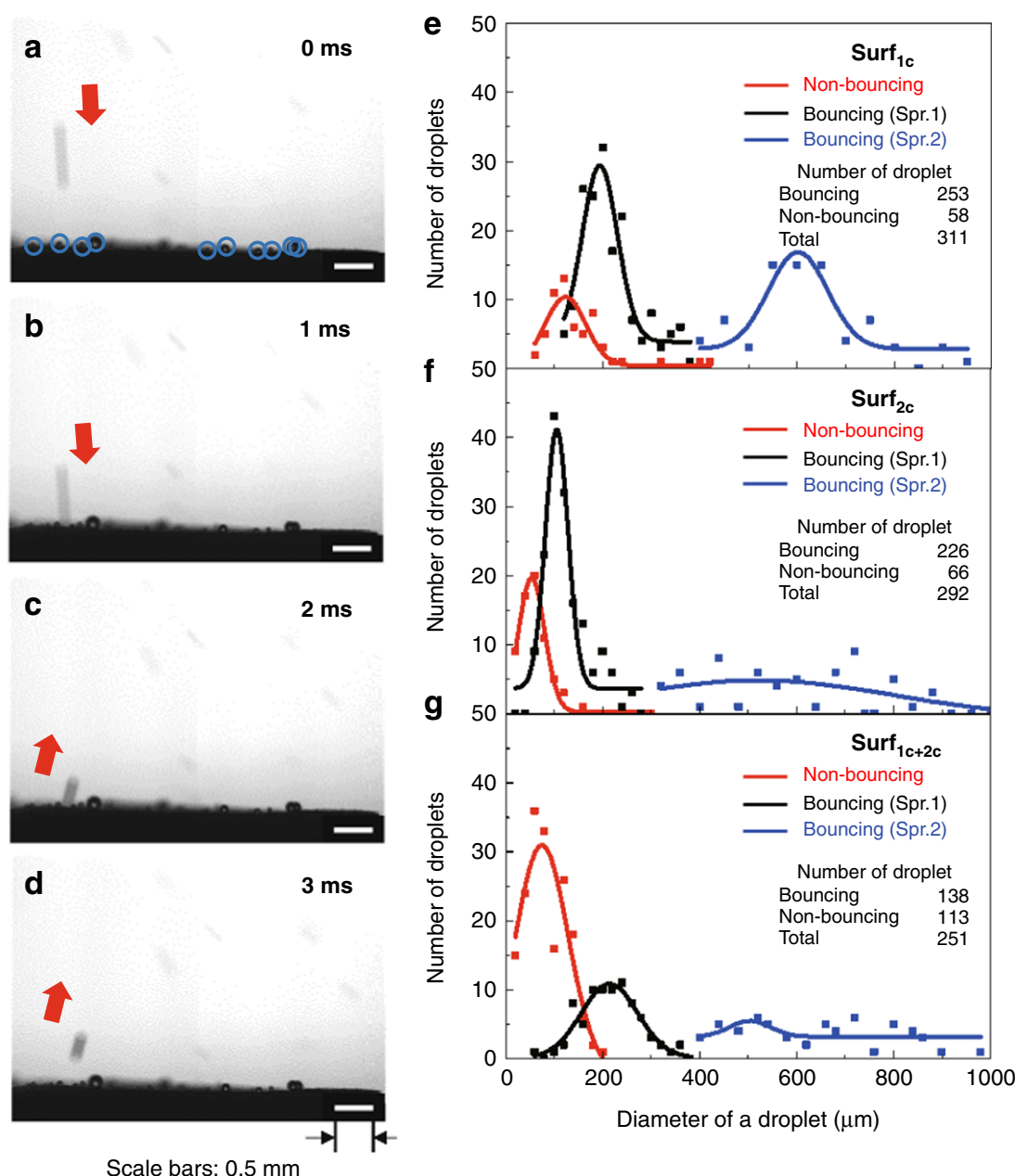

Fig. 5 Optical images of a microdroplet and size distributions. a-d Optical afterimages of the movement of a water droplet during bouncing on $\mathbf{S u r f} \mathbf{f}_{\mathbf{1 c}+\mathbf{2} \mathbf{c}}$ in $3 \mathrm{~ms}$. Red arrows indicate direction of droplet's movement. Blue circle marks are droplets adsorbing on the surface. e-g Distributions of diameters of water droplets contacted with Surf $_{\mathbf{1 c}}$, Surf $_{\mathbf{2 c}}$, and Surf $\mathbf{S u}_{\mathbf{1 c}+\mathbf{2 c}}$. e Distribution of diameters of water droplets contacted $\mathbf{S u r f}_{\mathbf{1 c}} \mathbf{f}_{\text {contacting }}$ Surf $\mathbf{2}_{\mathbf{2}}$, and $\mathbf{g}$ contacting

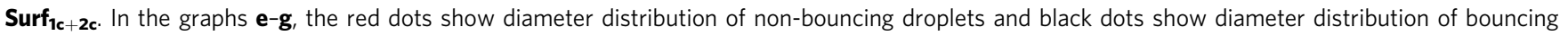
droplets generated by sprayer 1 (Spr. 1: Generating the microdroplet of 40-400 mm in diameter). The blue dots show diameter distribution of bouncing droplets generated by sprayer 2 (Spr. 2: generating the microdroplets of 400-1000 mm in diameter). Number of droplets used for preparation of distribution is indicated inside the graphs $\mathbf{e}-\mathbf{g}$. Scale bars for all optical images are $0.5 \mathrm{~mm}$ 
the bouncing and non-bouncing ranges are separated by mixing the two diarylethenes. For these distributions, the values of average droplet diameters are summarized in Table 1. On the surfaces of Surf $_{\mathbf{1 c}}$ and Surf $_{2 \mathrm{c}}$, the difference between the average size of a droplet showing bouncing and one showing non-bouncing behavior is relatively narrow, at 76.2 and $66.8 \mu \mathrm{m}$, respectively. On the other hand, this difference for Surf $_{1 \mathbf{c}+\mathbf{2 c}}$ was rather larger $(155.1 \mu \mathrm{m})$. These results indicate that by mixing the two diarylethene derivatives, the difference between bouncing and non-bouncing water droplets became dramatically separated; in other words, the dependence on the size of water droplets could be clearly distinguished for both types of wettability.

Furthermore, in order to clarify the significance of the size of droplets showing either the bouncing or non-bouncing phenomena, we fitted the sizes of real fog, drizzle and rain to the diameter distribution of water droplet contacting Surf $\mathbf{1 c}_{\mathbf{1 c}}$ +2 c. In 2017, S. Glienke and co-worker reported the quantification of the sizes of a cloud droplet (i.e., a fog droplet), a drizzle droplet, and a rain droplet with reference to a previous report and a meteorological definition (Fig. 6a) (American Meteorological Society, Glossary of Meteorology.

\begin{tabular}{|c|c|c|}
\hline & $\begin{array}{l}\text { Average diameter of } \\
\text { bouncing droplets }(\mu \mathrm{m})\end{array}$ & $\begin{array}{l}\text { Average diameter of } \\
\text { non-bouncing droplets }(\mu \mathrm{m})\end{array}$ \\
\hline $\begin{array}{l}\text { Surf }_{1 c} \\
\text { Surf }_{2 c} \\
\text { Surf }_{1 c+2 c}\end{array}$ & $\begin{array}{r}222.1 \pm 74.7 \\
126.8 \pm 48.9 \\
238.8 \pm 99.3\end{array}$ & $\begin{array}{r}145.9 \pm 68.5 \\
60 \pm 28.7 \\
83.7 \pm 40.5\end{array}$ \\
\hline
\end{tabular}

"Drizzle", (2016) Available online at http://glossary.ametsoc. org/wiki/Drizzle.) ${ }^{28,29}$. The water droplet classified as rain is generated by repeating the collision and coalescence of the cloud droplet in the raincloud. Consequently, it is classified differently depending on the size. A droplet of $40-100 \mu \mathrm{m}$ in diameter is classified as a "cloud (fog) droplet". By surveying the literature, the definition of the size of a drizzle droplet usually sets the lower limit at the diameter to $\sim 100 \mu \mathrm{m}$. Furthermore, the American Meteorological Society defined the size of drizzle as a type of precipitation consisting of water droplets $<500 \mu \mathrm{m}$ in diameter but larger than $100 \mathrm{~nm}$, with droplets larger than $500 \mu \mathrm{m}$ considered raindrops. Therefore, a droplet with a 100-500 $\mu \mathrm{m}$ diameter is classified as a "drizzle droplet", and a droplet with a diameter larger than $500 \mu \mathrm{m}$ is classified as a "rain droplet". Following the above guidelines, we fitted the distribution results to the sizes of rain (Fig. 6b). Consequently, the region of non-bouncing droplets was fitted to the region of fog droplet, whereas the region of bouncing droplets was fitted to the region of drizzle and rain droplet regions. These results indicate that a surface mimicking the structures of the termite wing can collect fogsized droplets and repel droplets as large as drizzle- and rain-sizeddroplets selectively (Fig. 6c). In addition, this distribution was related to the impacting velocity of a water droplet (Fig. 6d). The non-bouncing droplets collide with the surface with relatively low speed, on the other hand, the bouncing one collide with the surface with relatively high speed. This dual wettability showed by Surf $\mathbf{1 c}_{\mathbf{c}+\mathbf{2 c}}$ very closely reproduces the function of a termite wing. Therefore, we succeeded in regenerating the function of termite wings by mimicking their structure by using our CGT to the microcrystalline surface of a mixture of diarylethene 10 and 20. a

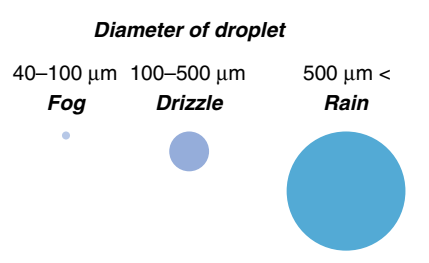

b

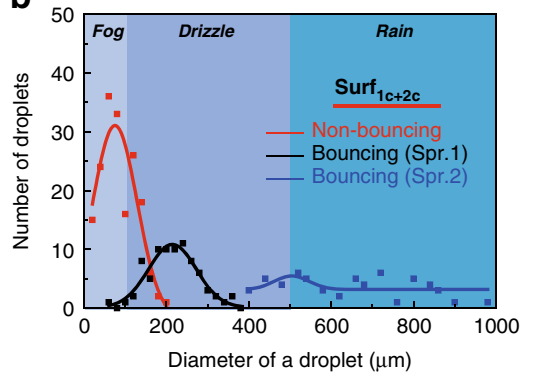

C

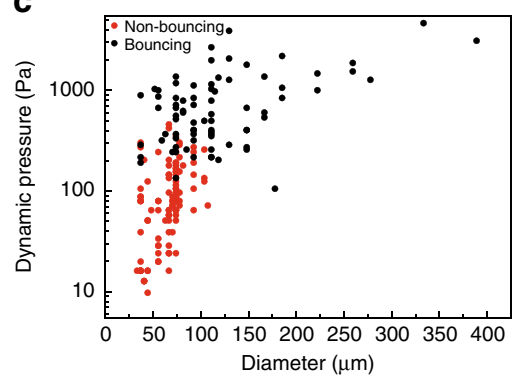

d

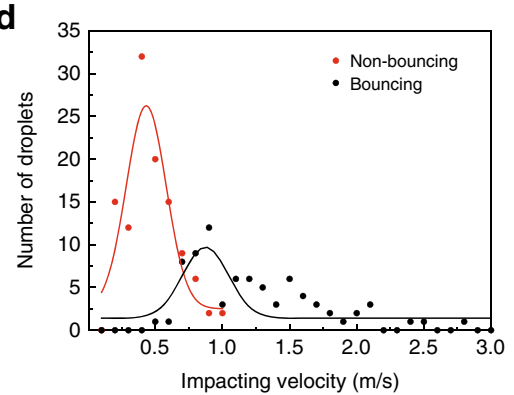

e

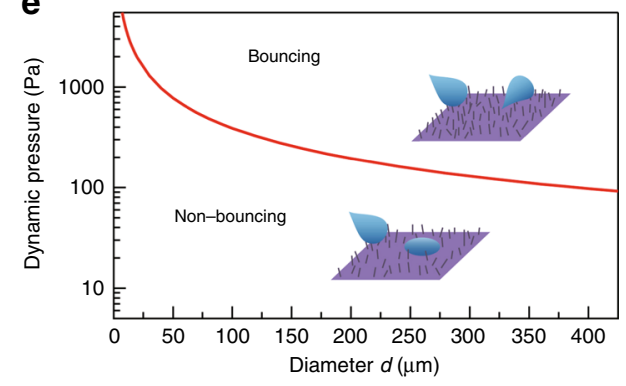

$\mathbf{f}$

Fig. 6 Classification and mechanical analysis of water droplets. a Classification by droplet's diameter. b Diameter distribution of bouncing (black) and nonbouncing (red) droplets matched to size regions of three types of rain. c Diameter-versus-dynamic pressure distribution of sprayed droplet. $\mathbf{d}$ The distribution of Impacting velocity of water droplets-versus-number of droplets. e Theoretical phase diagram of bouncing/non-bouncing behavior. Phase border is described by eq. (3) under the conditions of $f=0.088, e=0.62, \theta_{\text {flat }}=122.8^{\circ}$, and $\theta_{C B}=163.7^{\circ}$, where these values are obtained from the

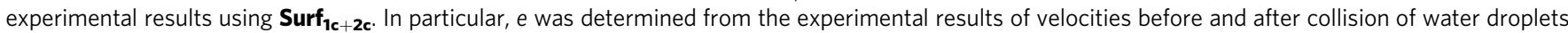
with different diameters. $\mathbf{f}$ Schematic illustration of bouncing and non-bouncing of microdroplets depending on droplet size 
a
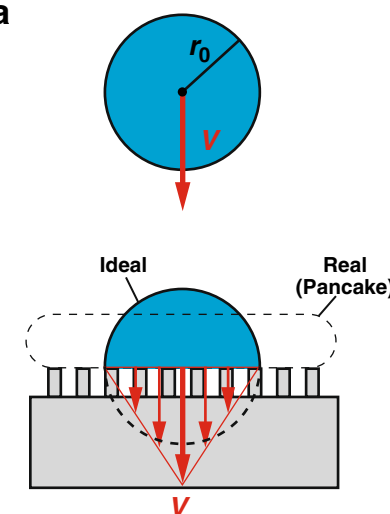

d

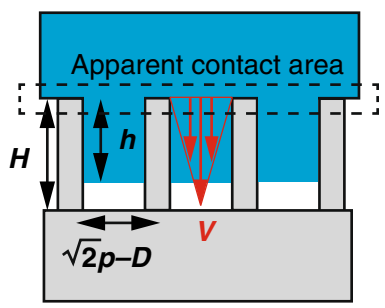

g

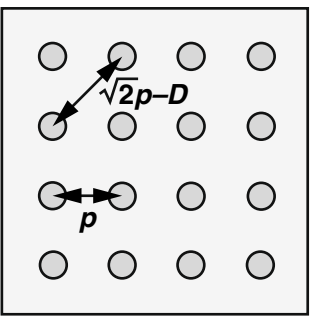

b

C

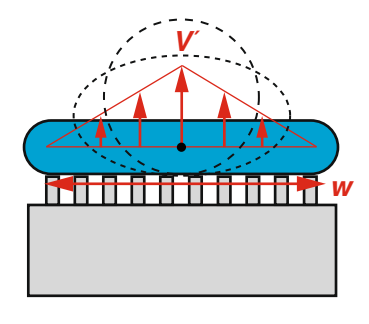

e

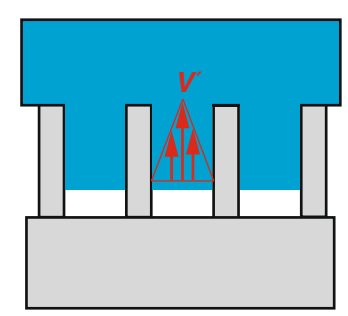

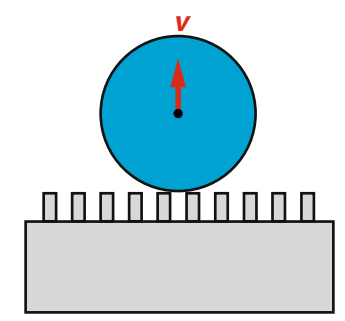

f

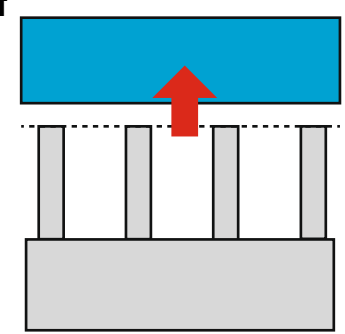

Fig. 7 Schematic representation of process of energy dissipation. a-c Dissipation energy in volume part, $\mathbf{d}-\mathbf{f}$ dissipation energy in the interfacial part, g geometry of surface structure (top view)

Theoretical explanation of dual wettability. To explain the phenomena theoretically, we measured the velocity at the time of collision onto the surface and the mass of all microdroplets from the optical images taken with a high-speed camera. We then calculated the dynamic pressures of impacting droplets, and compared these values with the Laplace pressure on the surface. We characterized collision velocity and mass and calculated the dynamic pressure for all droplet individually and then plotted the data to a graph (Fig. 6). The values of dynamic pressure $P_{d}$ were evaluated by Equation 1 ,

$$
P_{d}=\frac{1}{2} \rho V^{2}
$$

where $\rho$ is the density of water and $V$ is the collision velocity of a water droplet. In Fig. 6, the results of the non-bouncing droplets are represented by red dots, while those of the bouncing droplets are represented by black dots. According to the distribution, the two different phenomena were nearly switched at $\sim 200-300 \mathrm{~Pa}$. This result suggests that a threshold of dynamic pressure for bouncing phenomena exists on Surf $_{1 c+2 c}$.

To understand the threshold of dynamic pressure $P_{d}^{\text {th }}$, a simple theory is provided. Considering that the kinetic energy of a droplet after bouncing is equal to actual adhesion energy, $P_{d}^{\text {th }}$ can be formulated as

$$
P_{d}^{t h}=\frac{5.1 \gamma_{L}\left(1+\cos \theta_{\text {flat }}\right) f}{e^{2} d}
$$

where the factor of 5.1 is obtained from the apparent contact area of the droplet, $\gamma$ is surface tension of liquid (water), $e$ is the restitution coefficient, $\theta_{\text {flat }}$ is the equilibrium contact angle on a flat surface, $f$ is the area fraction of a solid surface in the Cassie-Baxter wetting state, and $d$ is the diameter of a droplet (see, Supplementary Information for the derivation of Equation 2). Figure 6e shows the theoretically obtained results of $P_{d}^{t h}$, which explain well Fig. 6c. The importance of adhesion energy to the bouncing ability of microdroplets is thus confirmed theoretically. Actually, $f, e$ and the state of the surfaces $\left(\right.$ Surf $_{\mathbf{1 c}}, \mathbf{S u r f}_{\mathbf{2 c}}$, and $\mathbf{S u r f}_{\mathbf{1 c}+\mathbf{2 c}}$ ) are strongly related to each other (see, Supplementary Information).

To understand the correlation between $f, e$ and the typical surface scales, we tried to describe $e$ of Surf $_{\mathbf{1 c}}, \mathbf{S u r f}_{\mathbf{2 c}}$, and Surf $_{1 \mathbf{1 c}+\mathbf{2 c}}$ in terms of $V$, average interval between needleshaped crystals and diameter. To discuss $e$, it is necessary to consider dissipation energy in bouncing droplets. Considering dissipation energy in volume and interfacial parts of microdroplets as shown in Fig. 7, residual energy of microdroplets after bouncing on Surf 1c $_{\mathbf{c}}$ and Surf $_{2 \mathrm{c}} E_{\text {res }}$ is

$$
\begin{gathered}
E_{\text {res }}=\frac{1}{2} m v^{2}=\frac{1}{2} m V^{2}-\frac{4 \pi \eta r_{0}^{2} V}{3}\left[1+\frac{4 r_{0}^{2} V^{\prime}}{w^{2} V}\right] \\
-\frac{\alpha \pi^{2} \eta r_{0}^{2} h^{2} V}{p^{2}}\left[1+\frac{V^{\prime}}{V}\right]-\alpha \pi r_{0}^{2} \gamma_{L}\left(1+\cos \theta_{\text {flat }}\right)\left(f+\frac{4 D h}{p^{2}}\right)
\end{gathered}
$$

where $(1 / 2) m v^{2}$ is kinetic energy corresponding to the residual energy after bouncing, $m$ is mass of microdroplets, $\eta$ is viscosity of water, $w$ is the width of wetting area as shown in 
a

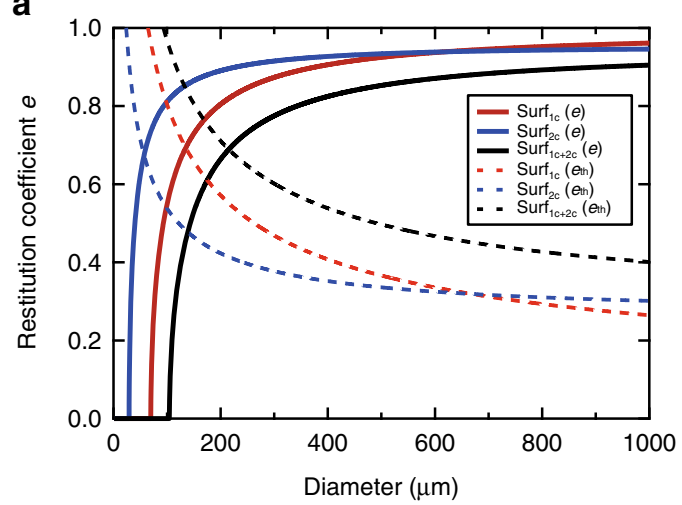

C

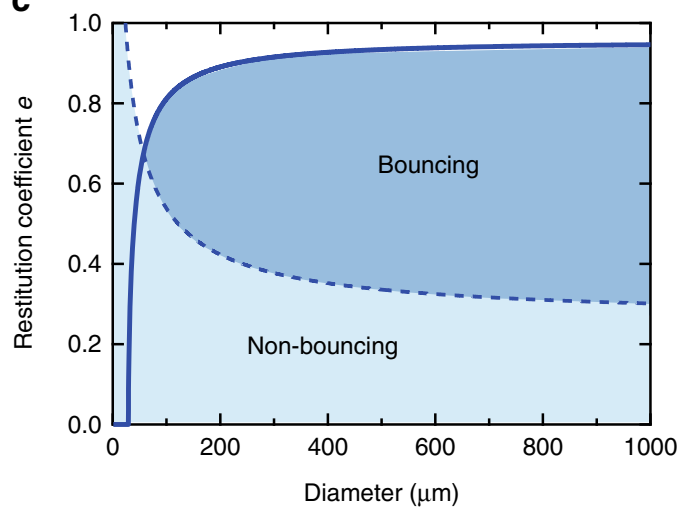

b

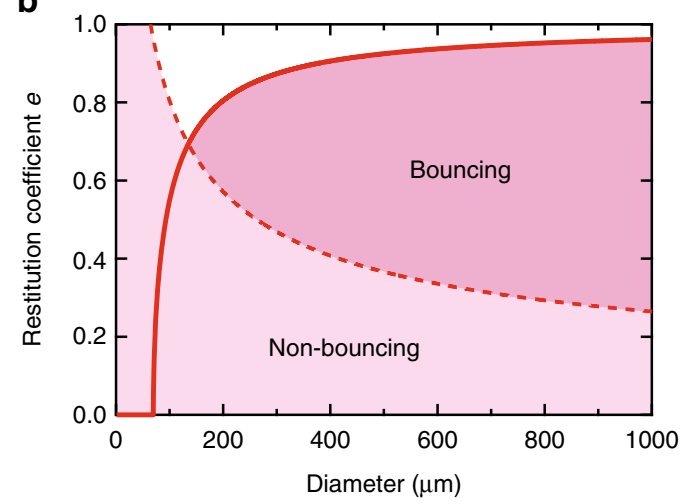

d

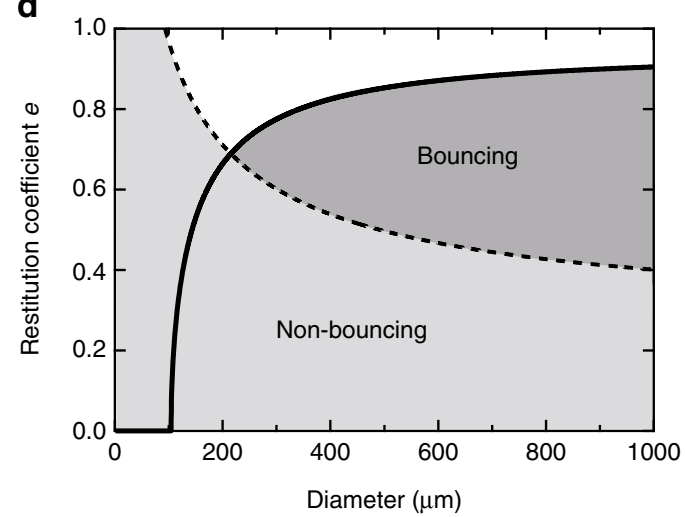

Fig. 8 Theoretical diagrams of bouncing/non-bouncing behaviors. a Dependences of restitution coefficients of Surf $_{\mathbf{1 c}}$, Surf $_{\mathbf{2} \mathbf{c}^{\prime}}$ and $\mathbf{S u r f}_{\mathbf{1 c}+\mathbf{2 c}}$ on diameter. b-d The phase diagrams of $\mathbf{S u r f}_{\mathbf{1} \mathbf{c}}, \mathbf{S u r f}_{\mathbf{2} \mathbf{c}}$, and Surf $_{\mathbf{1 c}+\mathbf{2} \mathbf{c}}$, respectively. The solid curves in a-d are restitution coefficient $e$, whereas the dotted curves are the thresholds of restitution coefficients $e_{t h}$. The areas highlighted by deep and light colors in $\mathbf{b}$-d mean the theoretical conditions of bouncing and non-bouncing, respectively. The conditions of calculations are shown in text

Fig. $7 \mathrm{~b}, \alpha$ is the ratio of wetting area in comparison with $\pi r_{0}^{2}, V^{\prime}$ is recoil velocity $\left(\left(V^{\prime}\right)^{2}=V^{2}-2 \eta V /\left(r_{0} \rho\right)-3 \alpha \pi \eta V h^{2} /\left(2 r_{0} \rho p^{2}\right)\right.$, $\rho$ : density of water), $p$ is the average interval between needleshaped crystals and $h$ is penetration depth $\left(h=\eta r_{0} V /\left(6 \gamma_{L}\right)\right)$. Surface structures are reflected in the third and last terms of Equation 3. Since squared restitution coefficient $e^{2}$ corresponds to $(v / V)^{2}$, e can be obtained from Equation 3.

$$
\begin{aligned}
e= & \left(1-\frac{2 \eta}{r_{0} \rho V}\left[1+\frac{4 r_{0}^{2} V^{\prime}}{w^{2} V}\right]-\frac{3 \alpha \pi \eta}{2 r_{0} \rho p^{2} V} h^{2}\left[1+\frac{V^{\prime}}{V}\right]\right. \\
& \left.-\frac{3 \alpha \gamma_{L}\left(1+\cos \theta_{\text {flat }}\right)}{2 r_{0} \rho V^{2}}\left(f+\frac{4 D h}{p^{2}}\right)\right)^{1 / 2}
\end{aligned}
$$

From the condition that $E_{\text {res }}$ is required to be larger than energy to pull water droplets off the surface, $e$ should be larger than threshold of restitution coefficient $e_{t h}$. The conditions of $e$ to bounce of water droplets with radius $r_{0}$ is

$$
e \geq e_{t h}=\left(\frac{3 \alpha \gamma\left(1+\cos \theta_{\text {flat }}\right)}{2 r_{0} \rho V^{2}}\left(f+\frac{4 D h}{p^{2}}\right)\right)^{1 / 2}
$$

Computational results of the dependence of $e$ on $d\left(=2 r_{0}\right)$ for Surf $_{1 c}$ and Surf 2 are shown in Fig. 8a-c. The calculation conditions are $\eta=10^{-3} \mathrm{~Pa} \cdot \mathrm{s}, \rho=10^{3} \mathrm{kgm}^{-3}, V=1.00 \mathrm{~ms}^{-1}, \alpha$ $=3.40, \theta_{\text {flat }}=130.8^{\circ}$ and $129.5^{\circ}, f=0.249$ and 0.0816 for Surf $\mathbf{1 c}$ and Surf $_{2 \mathfrak{c}}$, respectively. Theoretically, water droplets bounce in the conditions between the curves of $e$ and $e_{t h}$. Obviously, the computational results show that the diameter of the bouncing droplets on Surf ic $_{1 \mathrm{c}}$ is larger than that on Surf fc $_{\text {c Next, let }}$ us discuss dual wettability on $\operatorname{Surf}_{1 \mathbf{c}+\mathbf{2 c}}$. As Surf $_{\mathbf{1 c}+\mathbf{2 c}}$ is consisted of Surf $_{1 c}$ and Surf $_{2 c}$, dissipation energies on Surf $1 c$ and Surf $_{2 c}$ should be considered to understand the bouncing droplets

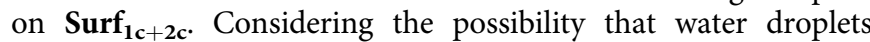
bounce on both the needle-shaped crystals of $1 \mathrm{c}$ and $2 \mathrm{c}$ for very short time or at the same time, then its dissipation energy is the sum of the dissipation energies on Surf 1 c and Surf Sc $_{\mathbf{1}}$. Then, residual energy on Surf $_{\mathbf{1 c}+\mathbf{2 c}} E_{r e s, \operatorname{Surf}_{1 c+2 c}}$ is obtained by subtracting dissipation energies of $\operatorname{Surf}_{\mathbf{1 c}}$ and $\operatorname{Surf}_{\mathbf{2 c}}$ from $(1 / 2) m V^{2}$.

$$
\begin{aligned}
E_{\text {res }, \text { Surf }_{1 c+2 c}}= & \frac{1}{2} m v^{2}=\frac{1}{2} m V^{2} \\
& -\left[\frac{4 \pi \eta r_{0}^{2} V}{3}\left(1+\frac{4 r_{0}^{2} V^{\prime}}{w^{2} V}\right)+\frac{\alpha \pi^{2} \eta r_{0}^{2} h^{2} V}{p^{2}}\left(1+\frac{V^{\prime}}{V}\right)\right. \\
& \left.+\alpha \gamma_{L}\left(1+\cos \theta_{\text {flat }}\right) \pi r_{0}^{2}\left(f+\frac{4 D h}{p^{2}}\right)\right]_{\operatorname{Surf}_{1 c}} \\
& -\left[\frac{4 \pi \eta r_{0}^{2} V}{3}\left(1+\frac{4 r_{0}^{2} V^{\prime}}{w^{2} V}\right)+\frac{\alpha \pi^{2} \eta r_{0}^{2} h^{2} V}{p^{2}}\left(1+\frac{V^{\prime}}{V}\right)\right. \\
& \left.+\alpha \gamma_{L}\left(1+\cos \theta_{\text {flat }}\right) \pi r_{0}^{2}\left(f+\frac{4 D h}{p^{2}}\right)\right]_{\operatorname{Surf}_{2 c}}
\end{aligned}
$$


a

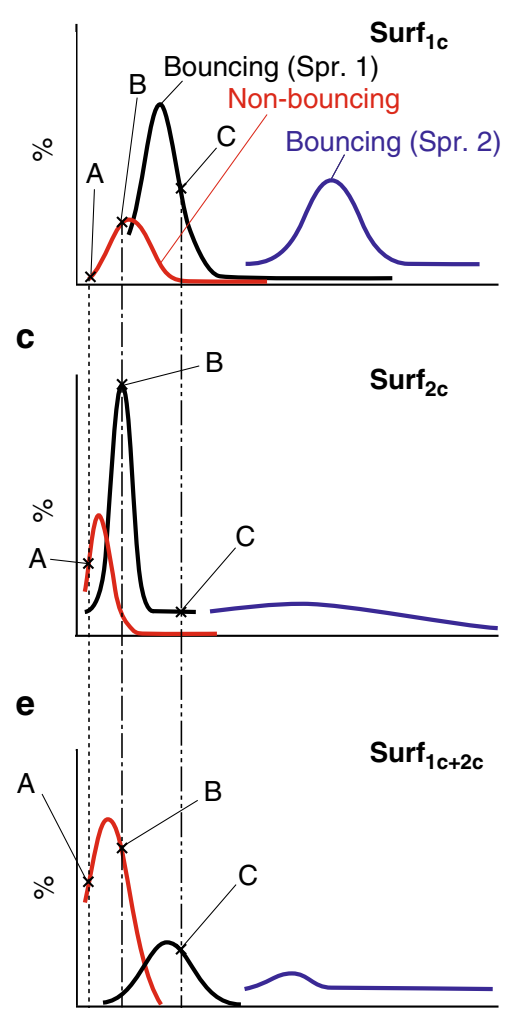

b

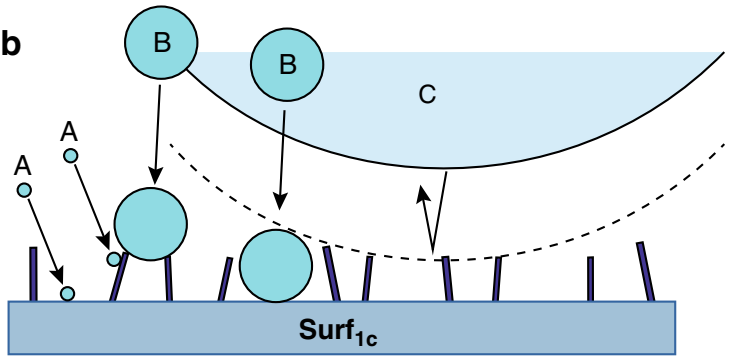

d

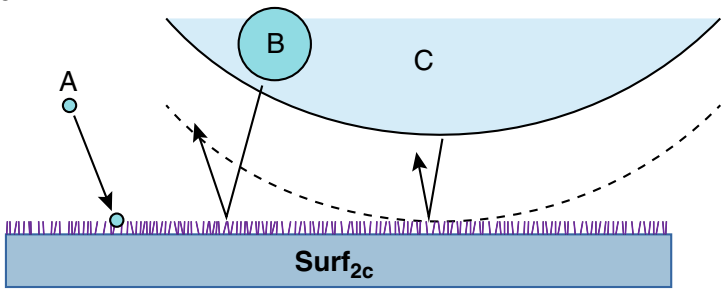

f

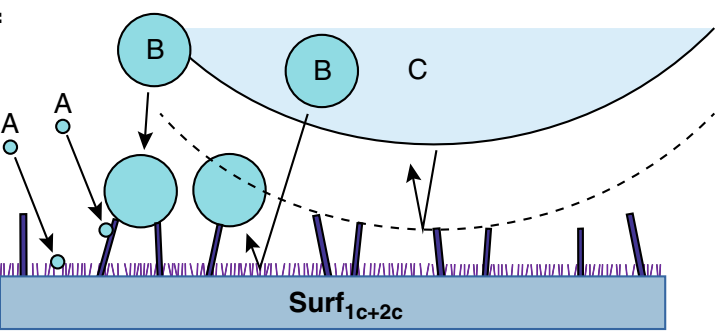

Fig. 9 Theoretical situation of bouncing droplet. a, c, e Schematic representations of the obtained distributions of bouncing and non-bouncing droplets of Surf $_{\mathbf{1} \mathbf{c}}$ Surf $_{\mathbf{2 c}}$, and Surf $_{\mathbf{1 c}+\mathbf{2} \mathbf{c}} \cdot \mathbf{b}, \mathbf{d}, \mathbf{f}$ Schematic representations of bouncing/non-bouncing behaviors suggested by theory. a-b Non-bouncing microdroplets (A) and bouncing microdroplets ( $B$ and $C$ ) shown by the crosses $(x)$ in the distributions of bouncing and non-bouncing on Surf $\mathbf{1}_{\mathbf{c}}$ in $\mathbf{a}$ are illustrated in b. $\mathbf{c}-\mathbf{d}$ The same on Surf $\mathbf{2 c}$ and $\mathbf{e - f}$ on Surf $\mathbf{1 c + 2 \mathbf { c }}$

Squared restitution coefficient of $\operatorname{Surf}_{\mathbf{1 c}+\mathbf{2 c}} e_{S u r f_{1 c+2 c}}^{2}$ is

$$
\begin{aligned}
e_{\text {Surf }_{1 c+2 c}}^{2}= & 1-\left[\frac{2 \eta}{r_{0} \rho V}\left(1+\frac{4 r_{0}^{2} V^{\prime}}{w^{2} V}\right)+\frac{3 \alpha \pi \eta}{2 r_{0} \rho p^{2} V} h^{2}\left(1+\frac{V^{\prime}}{V}\right)\right. \\
& \left.+\frac{3 \alpha \gamma_{L}\left(1+\cos \theta_{\text {flat }}\right)}{2 r_{0} \rho V^{2}}\left(f+\frac{4 D h}{p^{2}}\right)\right]_{\text {Surf }_{1 c}} \\
& -\left[\frac{2 \eta}{r_{0} \rho V}\left(1+\frac{4 r_{0}^{2} V^{\prime}}{w^{2} V}\right)+\frac{3 \alpha \pi \eta}{2 r_{0} \rho p^{2} V} h^{2}\left(1+\frac{V^{\prime}}{V}\right)\right. \\
& \left.+\frac{3 \alpha \gamma_{L}\left(1+\cos \theta_{\text {flat }}\right)}{2 r_{0} \rho V^{2}}\left(f+\frac{4 D h}{p^{2}}\right)\right]_{\text {surf }_{2 c}}
\end{aligned}
$$

The conditions to bounce on $\operatorname{Surf}_{\mathbf{1 c}+\mathbf{2 c}}$ is

$$
\begin{aligned}
e \geq e_{\text {th }}= & \left(\left[\frac{3 \alpha \gamma\left(1+\cos \theta_{\text {flat }}\right)}{2 r_{0} \rho V^{2}}\left(f+\frac{4 D h}{p^{2}}\right)\right]_{\text {Surf }_{1 c}}\right. \\
& \left.+\left[\frac{3 \alpha \gamma\left(1+\cos \theta_{\text {flat }}\right)}{2 r_{0} \rho V^{2}}\left(f+\frac{4 D h}{p^{2}}\right)\right]_{\text {Surf }_{2 c}}\right)^{1 / 2}
\end{aligned}
$$

Computational result of the dependence of $e$ on $r_{0}$ for $\operatorname{Surf}_{1 \mathrm{c}+\mathbf{2 c}}$ is shown in Figs. $8 \mathrm{a}$, d. The computational result shows that the diameter of the bouncing microdroplets on Surf $_{\mathbf{1 c}+\mathbf{2 c}}$ is larger than that of Surf $_{1 \mathbf{c}}$. The computational results in Fig. 8 thus reproduced the distributions of bouncing and non-bouncing droplets in Fig. 5e-g. Actually, a small droplet remains on Surf $_{1 \mathbf{c}+\mathbf{2 c}}$ as shown in Fig. $4 \mathrm{~d}$. This is not included in the theory, but it is caused by very long needle-shaped crystals of 1c; an example of such a very long needle-shaped crystal is observed in the left side of Fig. 3g. Figure 9 shows the schematic representations of bouncing and non-bouncing droplets based on Fig. 8 and it explains the reason why the obtained distribution of non-bouncing droplets is enhanced on Surf $\mathbf{1 c}_{\mathbf{1 c}+\mathbf{2 c}}$ than that on Surf $_{\mathbf{2 c}}$ and Surf 1c. On Surf $_{\mathbf{1 c}+\mathbf{2 c}}$, smaller droplets with bouncing size on Surf $_{2 c}$ are collected onto Surf ${ }_{1 c}$ efficiently. In addition, kinetic energy in bouncing larger droplets is dissipated, the residual energy decreases and the conditions of non-bouncing are amplified. Surface structure thus determines dissipation energy and bouncing behaviors of water droplets. Detail of the theory is described in Supplementary Discussion.

\section{Discussion}

In conclusion, microcrystalline films consisting of the mixtures of diarylethenes 10 and 20 were prepared as a first attempt to mimic the surface structure of a termite wing. The mixed microcrystalline film formed needle-shaped crystals of both $1 c$ and $2 c$ upon UV irradiation followed by storage above $T_{\mathrm{g}}$ in the dark. On Surf $\mathbf{f}_{1 \mathrm{c}}$, consisting of only larger needle-shaped crystals of 1c, water did not show bouncing phenomena, while on Surf fc $_{2}$ consisting of only smaller needle-shaped crystals of $\mathbf{2 c}$, clear bouncing behavior was 
observed. By mixing these two different sizes of needle-shaped crystals, simultaneous water-adhesive and water-bouncing phenomena were observed. These results indicate that larger crystals work well for adhesive force on the surface, while smaller-sized crystals work well for bouncing behavior. Furthermore, Surf $1 \mathbf{1 c + 2 c}$ demonstrated its ability to both capture fog-sized droplets and repel rain-sized droplets, which is determined by the size of droplet. Consequently, we succeeded in regenerating the surface function of the termite wing by using the photoinduced CGT for diarylethenes, and the dual wettability observed on Surf $_{\mathbf{1 c}+2 \mathbf{c}}$ was consistent with those on the wing surfaces of Nasutitermes $s p$. and Microcerotermes sp. Dual wettability of Surf $\mathbf{1 c + 2 c}$ was explained theoretically by dissipation energy in bouncing microdroplets and adhesion energy between microdroplets and Surf $\mathbf{f c}_{\mathbf{c}+2 \mathbf{c}}$. Such surface functions inspired by nature will lead to novel advanced materials, such as self-cleaning surfaces and parts for water collection and conservation systems. This study represents one of the research efforts made toward understanding how the surface of the termite wing, in nature, has effectively achieved the ability to apply both key properties of wetting.

\section{Methods}

General information. A SEM (KEYENCE VE-8800) was used to observe the microcrystalline surfaces of diarylethenes. A Xe lamp (Ushio 500-W) was used to irradaite the visible light with an optical cutoff filter (Toshiba color filter Y48; $\lambda>480 \mathrm{~nm}$ ). A hand lamp (Topcon UV lamp PU-21 (main $\lambda=254 \mathrm{~nm}, 23 \mathrm{~W}$ ) and Spectroline Hand-Held UV lamp, E-series $(\lambda=313 \mathrm{~nm}, 8 \mathrm{~W}))$ were used for UV light irradiation. The measurements of statistic and dinamic contact angles of the water droplets was monitored on Drop master 500 from Kyowa Interface Science Co., Ltd. by use of distilled water. The volume of water droplets was $1.5 \mu \mathrm{L}$. A micro slide glass plate from Matsunami Glass (Thickness 1.0 1.2 mm) was used for the subphase of diarylethene microcrystalline films.

Preparation and characterization of the film. The microcrystalline films were prepared by coating chloroform solutions containing $10\left(640 \mathrm{mg} \mathrm{mL}^{-1}\right), 2 \mathbf{o}(540$ $\left.\mathrm{mg} \mathrm{mL}{ }^{-1}\right)$, and a mixture of 10 and $20\left(540 \mathrm{mg} \mathrm{mL}^{-1}\right.$, with both compounds mixed in equimolar amount (1o: $243 \mathrm{mg}$; $4.46 \times 10^{-4} \mathrm{~mol}$ and 2o: $297 \mathrm{mg}$; $4.38 \times$ $10^{-4} \mathrm{~mol}$ )) onto glass substrates, and the solvent was evaporated in vacuo. A SEM (KEYENCE VK-8800) was used to observe the microstructure of the surfaces. The film thickness was $\sim 20 \mu \mathrm{m}$ from the SEM images taken from a side view of the microcrystalline film. Static CAs and SAs of the water droplets were measured with an optical contact angle meter (Kyowa Interface Science Co., Ltd., Drop Master $500)$. Visible light $(\lambda>500 \mathrm{~nm})$ irradiation was carried out using an Ushio $500-\mathrm{W}$ xenon lamp with a cutoff filter (Toshiba color filter Y-50), and UV light irradiation was carried out with a Spectroline Hand-Held UV lamp, E-series $(\lambda=254 \mathrm{~nm}, 820$ $\mathrm{mW} / \mathrm{cm}^{2}$ (distance: $15 \mathrm{~cm}$ )). Photoirradiation experiments at the eutectic temperature were carried out using a thermo-controller (FP82HT hot stage). The crystal data were already reported in a previous paper $^{2}$. For the fractal analysis, microcrystalline film samples on the glass substrate were set on the electron microscope's stage with conductive carbon tape, and the Au-Pd alloy was evaporated onto the sample surface. The cross-section was observed using a sample that was cracked together with the cover glass and set perpendicularly on the stage.

Fractal analysis of films. The fractal dimension of the cross-section of the rough solid surfaces was evaluated from the trace curves of the surfaces by the boxcounting method. A two-dimensional space containing the trace curve was divided by identical boxes of side size $r$ like the cross-section of a piece of paper. The number of boxes, containing trace curve $N(r)$ was counted for use as a function of side size $r$. Based on this box-counting method, the fractal dimension can be obtained from the following relationship:

$$
N(r) \propto r^{-D}
$$

where $D$ is the fractal dimension. The dimension of surface $D_{\mathrm{s}}$ is approximately obtained by $D_{\mathrm{s}}=D+1^{30}$.

Fractal analysis of the microcrystalline surface was carried out by the boxcounting method (Supplementary Fig. 13). As shown in Supplementary Fig. 13a, for the mixture of $\mathbf{1 0}$ and $\mathbf{2 0}$, there are two fractal regions. The smaller region from 0.11 to $5.16 \mu \mathrm{m}$ is attributed to the needle-shaped crystals of $\mathbf{2 c}$, whereas the larger region from 5.16 to $29.1 \mu \mathrm{m}$ is attributed to the needle-shaped crystals of $\mathbf{1 c}$. On the Surf $_{1 c}$, the fractal region expanded from 0.57 to $30.4 \mu \mathrm{m}$, whereas on the Surf ${ }_{2 \mathrm{c}}$ this region expanded from 0.21 to $5.6 \mu \mathrm{m}$. Although the fractal dimensions $(D \mathrm{~s})$ of these regions are very similar to each other around 2.4 , the needle-shaped crystals of both $1 \mathrm{c}$ and $2 \mathrm{c}$ shifted to smaller sizes.

For example, the smallest size of the fractal region of mixed film was $0.11 \mu \mathrm{m}$, which is attributable to the diameter of the needle-shaped crystal of $2 \mathrm{c}$, is smaller than that of $2 \mathbf{o}$ film $(0.21 \mu \mathrm{m})$, and the largest size of that of the mixed film of 29.1 $\mu \mathrm{m}$, which is attributable to the largest diagonal size of $\mathbf{1 c}$ crystal, is also smaller than that of 10 film $(30.4 \mu \mathrm{m})$. During the crystal growths of the needle-shaped crystals of $1 \mathbf{c}$ and $2 \mathrm{c}$, the self-aggregation process may be only slightly disturbed by additional components of each other.

\section{Data availability}

The authors declare that all data supporting the findings of this study are available within the article and its supplementary information files.

Received: 10 March 2019 Accepted: 8 July 2019

Published online: 06 August 2019

\section{References}

1. Feng, L. et al. Super-hydrophobic surfaces: from natural to artificial. Adv. Mater. 14, 1857-1860 (2002).

2. Shi, F. et al. Towards understanding why a superhydrophobic coating is needed by water striders. Adv. Mater. 19, 2257-2261 (2007).

3. Lee, H., Lee, B. P. \& Messersmith, P. B. A reversible wet/dry adhesive inspired by mussels and geckos. Nature 448, 338-341 (2007).

4. Zi, J. et al. Coloration strategies in peacock feathers. Proc. Natl. Acad. Sci. USA 100, 12576-12578 (2000)

5. Xu, D., Yu, H., Xu, Q., Xu, G. \& Wang, K. Thermoresponsive photonic crystal: synergistic effect of poly(N-isopropylacrylamide)-co-acrylic acid and morpho butterfly wing. ACS Appl. Mater. Interfaces 7, 8750-8756 (2015).

6. Pris, D. et al. Towards high-speed imaging of infrared photons with bioinspired nanoarchitectures. Nat. Photonics 6, 195-200 (2012).

7. Li, X.-M., Reinhoudt, D. \& Calama, M. C. What do we need for a superhydrophobic surface? A review on the recent progress in the preparation of superhydrophobic surfaces. Chem. Soc. Rev. 36, 1350-1368 (2007).

8. Zhang, X., Shi, F., Niu, J., Jiang, Y. \& Wang, Z. Superhydrophobic surfaces: from structural control to functional application. J. Mater. Chem. 18, 621-633 (2008)

9. Lee, Y., Park, S.-H., Kim, K.-B. \& Lee, J.-K. Fabrication of hierarchical structures on a polymer surface to mimic natural superhydrophobic surfaces. Adv. Mater. 19, 2330-2335 (2007).

10. Parker, R. \& Townley, H. E. Biomimetics of photonic nanostructures. Nat. Nanotechnol. 2, 347-353 (2007).

11. Zhou, L. Bio-inspired study of structural materials. Mater. Sci. Eng. C. 11, $13-18(2000)$

12. Xia, F. \& Jiang, L. Bio-inspired, smart, multiscale interfacial materials. $A d v$. Mater. 20, 2842-2858 (2008).

13. Barthlott, W. \& Neinhuis, C. Purity of the sacred lotus, or escape from contamination in biological surfaces. Planta 202, 1-8 (1997).

14. Neinhuis, C. \& Barthlott, W. Characterization and distribution of waterrepellent, self-cleaning plant surfaces. Ann. Bot. 79, 667-677 (1997).

15. Tian, Y., Su, B. \& Jiang, L. Interfacial material system exhibiting superwettability. Adv. Mater. 26, 6872-6897 (2014).

16. Holdgate, M. W. The wetting of insect cuticles by water. J. Exp. Biol. 32, 591-617 (1955).

17. Wagner, P., Neinhuis, C. \& Barthlott, W. Wettability and contaminability of insect wings as a function of their surface sculptures. Acta Zool. 77, 213-225 (1996).

18. Watson, G. S., Cribb, B. W. \& Watson, J. A. How micro/nanoarchitecture facilitates anti-wetting: an elegant hierarchical design on the termite wing. ACS Nano 4, 129-136 (2010).

19. Watson, G. S., Cribb, B. W. \& Watson, J. A. Contrasting micro/nano architecture on termite wings: two divergent strategies for optimising success of colonisation flights. Plos ONE 6, e124368 (2011).

20. M. J. Pearce. Termites biology and pest management; CAB International: UK p 172 (1997).

21. Uchida, K. et al. Photoinduced reversible formation of microfibrils on a photochromic diarylethene microcrystalline surface. Angew. Chem. Int. Ed. 45, 6470-6473 (2006).

22. Uchida, K. et al. Phototunable diarylethene microcrystalline surfaces: lotus and petal effects upon wetting. Angew. Chem. Int. Ed. 49, 5942-5944 (2010).

23. Nishikawa, N. et al. Photoinduced formation of superhydrophobic surface on which contact angle of a water droplet exceeds $170^{\circ}$ by reversible topographical changes on a diarylethene microcrystalline surface. Langmuir 28, 17817-17824 (2012) 
24. Nishikawa, N. et al. Photoinduced self-epitaxial crystal growth of a diarylethene derivative with antireflection moth-eye and superhydrophobic lotus effects. Langmuir 29, 8164-8169 (2013).

25. Nishimura, R. et al. Fractal surfaces of molecular crystals mimicking lotus leaf with phototunable double roughness structures. J. Am. Chem. Soc. 138, 10299-10303 (2016).

26. Fujinaga, N. et al. Photoinduced topographical changes on microcrystalline surfaces of diarylethenes. CrystEngComm 18, 7229-7235 (2016).

27. Uchida, K., Nishimura, R., Hatano, E., Mayama, H. \& Yokojima, S. Photochromic crystalline systems mimicking bio-functions. Chem. Eur. J. 24, 8491-8506 (2018).

28. Glienke, S. et al. Cloud droplets to drizzle: contribution of transition drops to microphysical and optical properties of marine stratocumulus clouds. Geophys. Res. Lett. 15, 8002-8010 (2017).

29. Takahashi, T. Measurement of electric charge of cloud droplets, drizzle, and raindrops. Rev. Geophys. 4, 903-924 (1973)

30. H. Takayasu. Fractals in the physical sciences, Manchester University Press (1990).

\section{Acknowledgements}

We thank Dr. Wakako Ohmura (Department of Wood Improvement, Forestry and Forest Products Research Institute), Prof. Tsuyoshi Yoshimura (Laboratory of Innovative Humano-habitability, Kyoto University), Mr. Toyokazu Tanaka and Mr. Yusuke Saito (Duskin Co., Ltd.) for fruitful discussion about the termites and termite wings. This work was supported by JSPS KAKENHI Grant Number JP26107012 in Scientific Research on Innovative Areas "Photosynergetics", the CREST program (JPMJCR17N2) of the Japan Science and Technology Agency, and JSPS KAKENHI Grant Number JP26400424 and JP18K03554 in Scientific Research (C), JSPS KAKENHI Grant Number JP18J20078 in JSPS Research Fellow and the Ministry of Education, Culture, Sports, Science and Technology, Japan (MEXT) as a Supported Program for the Strategic Research Foundation at Private Universities.

\section{Author contribution}

R.N. and K.U. designed and prepared the microcrystalline surface mimicking the surface structures of termite wing. R.N., H.M. and S.Y. explained the phenomena of bouncing and non-bouncing of water. R.N., K.H., H.M., S.Y., S.N. and K.U. discussed the obtained results and writing the manuscript.

\section{Additional information}

Supplementary information accompanies this paper at https://doi.org/10.1038/s42004019-0192-6.

Competing interests: The authors declare no competing interests.

Reprints and permission information is available online at http://npg.nature.com/ reprintsandpermissions/

Publisher's note: Springer Nature remains neutral with regard to jurisdictional claims in published maps and institutional affiliations.

(c) (i) Open Access This article is licensed under a Creative Commons Attribution 4.0 International License, which permits use, sharing, adaptation, distribution and reproduction in any medium or format, as long as you give appropriate credit to the original author(s) and the source, provide a link to the Creative Commons license, and indicate if changes were made. The images or other third party material in this article are included in the article's Creative Commons license, unless indicated otherwise in a credit line to the material. If material is not included in the article's Creative Commons license and your intended use is not permitted by statutory regulation or exceeds the permitted use, you will need to obtain permission directly from the copyright holder. To view a copy of this license, visit http://creativecommons.org/licenses/by/4.0/.

(C) The Author(s) 2019 